\title{
Joint Digital Self-interference Cancellation in Full-duplex Radios under IQ Imbalance and Transmitter Non-linearity
}

\author{
Meijing Zhou ${ }^{1 *}$, Nan Chen ${ }^{1}$, Changhua Zhu ${ }^{1}$, and Yunhui $\mathrm{Yi}^{1}$ \\ ${ }^{1}$ State Key Laboratory of Integrated Services Networks, Xidian University, Xi' an, Shaanxi, China
}

\begin{abstract}
RF imperfections can significantly degrade the performance of full-duplex wireless communication system by introducing non-idealities and random effects, which make it difficult to cancel the self-interference completely. In this paper, we first address the adverse benefits of both the transmitter non-linearity and the IQ imbalance. Then on the basis of these, a joint digital self-interference cancellation scheme is proposed, in which not only the effect of IQ imbalance and power amplifier non-linearity individually, but also the comprehensive function of them are taken into account. Furthermore, the simulation is implemented in the MATLAB platform using standard WiFi 802.11ac PHYs. The results show that the proposed canceller can eliminate more compared with other cancellation schemes, and the overall self-interference attenuation can attain $108 \mathrm{~dB}$, which makes the residual self-interference closer to the noise floor.
\end{abstract}

\section{Introduction}

Recently, full-duplex communication has become one of the research hotspots in the field of wireless communication [1-3], since it can increase the capacity of the communication system theoretically $[4,5]$. However, the key problem in achieving a full-duplex wireless communication is the existence of strong self-interference (SI) signal.

At present, the commonly used method of SI cancellation is a combination of antenna separation, analog cancellation and digital cancellation [7-12]. However, the experimental data in $[2,3,5]$ show that it's impossible to realize the complete SI elimination in practical full-duplex system by only classical linear SI cancellation. This is mainly affected by the RF impairments in the transceiver chains, such as: transmitter and receiver non-linearity, IQ imbalance, oscillator phase noise, quantization noise and receiver Gaussian noise, etc. The strength of each noise source at different received SI signal has been shown in [13]: The nonlinear distortion is the main limiting factor. It's pointed out that the mirror component caused by IQ imbalance in the direct-conversion transceiver chains is generally $25-40 \mathrm{~dB}$ [6, $14,15]$. This means that the effect of IQ imbalance is not negligible, and must be taken into consideration.

In this paper, we first take both IQ imbalance and power amplifier (PA) non-linearity into account in the design of SI canceller for a 16QAM-based full-duplex radio. Based on

*Corresponding author: mjzhouxd@163.com 
the discussion of the IQ imbalance and transmitter non-linearity in digital domain, we proposed a joint cancellation scheme, which can eliminate not only the image component and PA nonlinear distortion, but also the combination of the two items. Moreover, the unknown parameters are estimated according to the least-square (LS) criterion. Finally, the performances of the proposed scheme together with other three SI cancellers in digital domain are compared, and the results show that the residual SI in our scheme is minimal and closest to the noise floor.

The remainder of the paper is organized as follows. In section 2, the structure of the considered full-duplex radios is presented, alongside with analysis of RF impairments. Then we propose a joint digital SI cancellation scheme in section 3. In section 4, the entire system is simulated and the performances of different algorithms are evaluated. Finally, the conclusions are drawn in section 5 .

\section{System model and RF impairments}

A full-duplex transceiver with SI cancellation is depicted in Fig. 1, where dual antennas are installed with distance of $20 \mathrm{~cm}$ [5]. Physical isolation is used to maximize the attenuation of SI in antenna separation, and here the antenna cancellation is assumed to be $25 \mathrm{~dB}$. As to analog cancellation, it's just assumed that the amount of SI cancellation is $30 \mathrm{~dB}$ according to the typical cancellation scheme in [7] since the emphasis of this paper is placed on the cancellation of the effects introduced by RF impairments in digital domain.

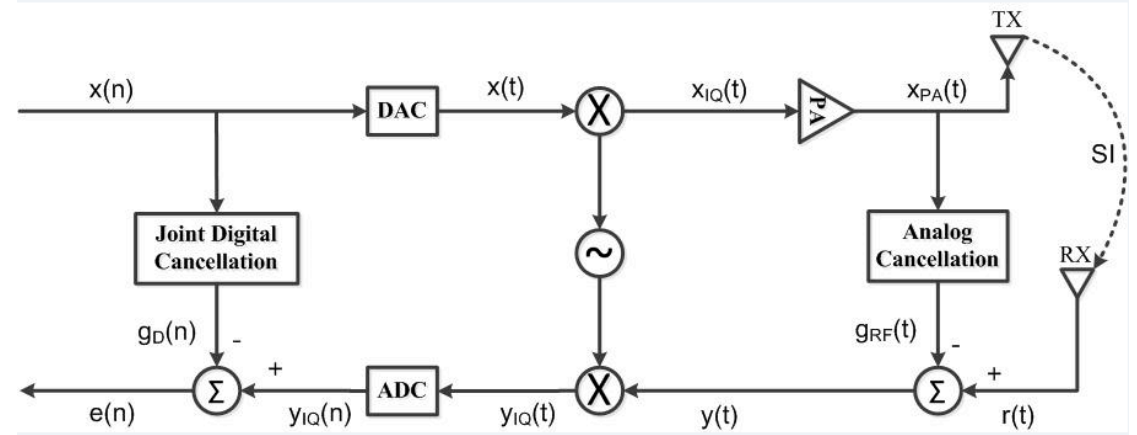

Fig. 1. A block of diagram for full-duplex transceiver with SI cancellation

In practical systems, it can't be negligible that each component of the transceiver is nonideal, so we must take the impairments of these components into account. According to the previous discussion, we mainly consider the effect of IQ imbalance and PA nonlinear distortion. And in order to make the model simple and convenient, other RF impairments are ignored temporarily in this paper.

\subsection{IQ imbalance}

IQ imbalance is a prominent problem in most of the transceiver chains, which is mainly caused by the mismatch between I and Q branches of the quadrature modulator. The ideal quadrature modulator generally has a constant gain and a linear phase, thus the gain of I and Q branches is equal, and also the phase of the local oscillator signal is orthogonal [16]. However, in practical circuit, the quadrature modulator always has a certain amplitude and phase deviation, which leads to the imbalance of the gain in I and Q branches, and finally results in the mirror-frequency interference. The structure of quadrature modulator with IQ imbalance is presented in Fig. 2. 
The signal $x(t)$ is up-converted into the signal at desired medium frequency by quadrature modulator, i.e. $x_{I}(t)$ and $x_{Q}(t)$, which represent the in-phase signal and quadrature-phase signal respectively. The symbol $k$ and $\phi$ in Fig. 2 represent the imbalance of amplitude and phase in local oscillator. Then the signal $y(t)$ can be written as

$$
\begin{aligned}
y(t) & =x_{I}(t) \cdot \cos (2 \pi f t)+x_{Q}(t) \cdot(1+k) \sin (2 \pi f t+\phi) \\
& =g_{1}(t) \otimes x(t)+g_{2}(t) \otimes x^{*}(t)
\end{aligned}
$$

where $g_{1}(t)$ is the response for the direct signal component, and $g_{2}(t)$ is the response for the image component [15]. Here $\otimes$ indicates the convolution operation, and $(\cdot)^{*}$ denotes the complex conjugate. The performance of quadrature modulator can be defined as image rejection ratio (IRR), and the expression of IRR is

$$
\operatorname{IRR}(d B)=10 \log 10 \frac{1+(1+\mathrm{k})^{2}+2(1+k) \cos \phi}{1+(1+k)^{2}-2(1+k) \cos \phi}=10 \log 10\left(\left|G_{1}(f)\right|^{2} /\left|G_{2}(f)\right|^{2}\right)
$$

where $G_{1}(f)$ and $G_{2}(f)$ are the Fourier transformation of $g_{1}(t)$ and $g_{2}(t)$ respectively.

\subsection{PA non-linearity}

The non-linearity of PA in transmitter is the most widely researched source of distortion in the existing literature. We use Taylor series expansion to approximate the non-linear function, which is a standard way to model non-linear functions [7]. Generally, the output of the nonlinear PA block can be written as

$$
y(t)=\sum_{m=1}^{M} a_{m} x^{m}
$$

It can be shown that for practical wireless systems, only the odd orders of the polynomial contribute to the in-band distortion [9], and the third order item is the main influence factor, so the higher orders are ignored in this paper. Thus we can rewrite (3) as

$$
y(t)=a_{1} x(t)+a_{3} x^{3}(t)
$$

where $a_{1}$ and $a_{3}$ are the weights of linear item and third order item respectively.

\subsection{SI cancellation model}

Based on the above analysis, the following cancellation model of the whole system only considers the PA non-linearity and IQ imbalance. According to the research in literature [9], we make some simplification to the analysis procedure, and expand the final SI signal model with the cross combination items of IQ imbalance and PA non-linearity.

The baseband signal is represented by $x(n)$, and $x(t)$ is the corresponding RF signal after digital-to-analog converter (DAC). The signal $x(t)$ is then up-converted into the required frequency for transmission by quadrature modulator with the error $k$ and $\phi$. Then according to (1), we can obtain the up-converted signal $x_{I Q}(t)$

$$
x_{I Q}(t)=g_{1, T X}(t) \otimes x(t)+g_{2, T X}(t) \otimes x^{*}(t)
$$

where $g_{1, T X}(t)$ and $g_{2, T X}(t)$ are the response of each item in the transmitter chain.

It should be amplified by PA in transmitter to the specified power before transmitting. The output of PA can be acquired according to (4)

$$
x_{P A}(t)=a_{1} x_{I Q}(t)+a_{3} x_{I Q}^{3}(t)
$$

For simplicity, $x_{I Q 3}(t)$ is used instead of $x_{I Q}^{3}(t)$ in (6), which refers to the third order non-linearity. As mentioned in section 2.2, the transmission path and analog canceller are 
regarded as a whole channel here, and $h_{R F}(t)$ is the response of the channel. So the signal before the quadrature modulator in $\mathrm{RX}$ radios can be written as

$$
y(t)=h_{R F}(t) \otimes x_{P A}(t)
$$

Similar to the transmitter chain, the signal is down-converted to the baseband frequency through quadrature modulator in the receiver chain, which also produces the mirrorfrequency interference. The equation for $y_{I Q}(t)$ is as follows

$$
y_{I Q}(t)=g_{1, R X}(t) \otimes y(t)+g_{2, R X}(t) \otimes y^{*}(t)
$$

where $g_{1, R X}(t)$ and $g_{2, R X}(t)$ are the response of each item in the receiver chain. Finally, the signal is digitized by analog-to-digital converter (ADC), $y_{I Q}(n)$ is the expression of $y_{I Q}(t)$ in digital domain.

To obtain the final digital cancellation model, we substitute (5) to (6), then take the results into (7), (8) successively. The signal $y_{I Q}(n)$ can be written as

$$
y_{I Q}(n)=h_{1}(n) \otimes x(n)+h_{2}(n) \otimes x^{*}(n)+h_{I Q 3}(n) \otimes x_{I Q 3}(n)+h_{I Q 3, i m}(n) \otimes x_{I Q 3}^{*}(n)+w(n)
$$

where $w(n)$ is the overall other noise in the transceiver, and $h_{1}(n), h_{2}(n), h_{I Q 3}(n)$ as well as $h_{I Q 3, i m}(n)$ represent the channel response of $x(n), x^{*}(n), x_{I Q 3}(n)$ and $x_{I Q 3}^{*}(n)$ respectively, they can be expanded as

$$
\begin{gathered}
h_{1}(n)=a_{1} g_{1, T X}(n) \otimes h_{R F}(n) \otimes g_{1, R X}(n)+a_{1}^{*} g_{2, T X}^{*}(n) \otimes h_{R F}^{*}(n) \otimes g_{2, R X}(n) \\
h_{2}(n)=a_{1} g_{2, T X}(n) \otimes h_{R F}(n) \otimes g_{1, R X}(n)+a_{1}^{*} g_{1, T X}^{*}(n) \otimes h_{R F}^{*}(n) \otimes g_{2, R X}(n) \\
h_{I Q 3}(n)=a_{3} h_{R F}(n) \otimes g_{1, R X}(n) \\
h_{I Q 3, i m}(n)=a_{3}^{*} h_{R F}^{*}(n) \otimes g_{2, R X}(n)
\end{gathered}
$$

From (9), it can be found that the total SI in the digital domain contains not only the linear components and the image components, but also the items $x_{I Q 3}(n)$ and $x_{I Q 3}^{*}(n)$, which are the cross combination items of IQ imbalance and PA nonlinear distortion. However, $x_{I Q 3}(n)$ and $x_{I Q 3}^{*}(n)$ are expressions about $x(n), x^{*}(n)$, and can't be obtained directly from the transmitted signal, so it's necessary to expand the two items continuously.

Following the polynomial expansion formula $(a+b)^{3}=a^{3}+3 a^{2} b+3 a b^{2}+b^{3}, x_{I Q 3}(n)$ and $x_{I Q 3}^{*}(n)$ can be written as

$$
\begin{aligned}
x_{I Q 3}(n) & =\left[g_{1, T X}(n) \otimes x(n)+g_{2, T X}(n) \otimes x^{*}(n)\right]^{3} \\
& =a_{1}(n) \otimes x^{3}(n)+3 a_{2}(n) \otimes\left[x^{2}(n) x^{*}(n)\right]+3 a_{3}(n) \otimes\left[x(n)\left(x^{*}(n)\right)^{2}\right]+a_{4}(n) \otimes\left(x^{*}(n)\right)^{3} \\
x_{I Q 3}^{*}(n) & =\left[\left(g_{1, T X}(n) \otimes x(n)+g_{2, T X}(n) \otimes x^{*}(n)\right)^{3}\right]^{*} \\
& =a_{4}^{*}(n) \otimes x^{3}(n)+3 a_{3}^{*}(n) \otimes\left[x^{2}(n) x^{*}(n)\right]+3 a_{2}^{*}(n) \otimes\left[x(n)\left(x^{*}(n)\right)^{2}\right]+a_{1}^{*}(n) \otimes\left(x^{*}(n)\right)^{3}
\end{aligned}
$$


where $a_{1}(n) \sim a_{4}(n)$ and $a_{1}^{*}(n) \sim a_{4}^{*}(n)$ are the weights of individual signal components. The signal $y_{I Q}(n)$ can be simply rewritten by substituting (14), (15) to (9) as

$$
\begin{aligned}
y_{I Q}(n) & =h_{1}(n) \otimes x(n)+h_{2}(n) \otimes x^{*}(n)+h_{3}(n) \otimes x^{3}(n)+h_{4}(n) \otimes\left(x^{*}(n)\right)^{3} \\
& +h_{5}(n) \otimes\left(x^{2}(n) x^{*}(n)\right)+h_{6}(n) \otimes\left(x(n)\left(x^{*}(n)\right)^{2}\right)+w(n)
\end{aligned}
$$

where $h_{1}(n)$ and $h_{2}(n)$ are the same as which in $(9), h_{3}(n) \sim h_{6}(n)$ are the response for the corresponding signal components, we can expand them as follows

$$
\begin{gathered}
h_{3}(n)=h_{I Q 3}(t) \otimes a_{1}(n)+h_{I Q 3, i m}(t) \otimes a_{4}^{*}(n) \\
h_{4}(n)=h_{I Q 3}(t) \otimes a_{4}(n)+h_{I Q 3, i m}(t) \otimes a_{1}^{*}(n) \\
h_{5}(n)=3 h_{I Q 3}(t) \otimes a_{2}(n)+3 h_{I Q 3, i m}(t) \otimes a_{3}^{*}(n) \\
h_{6}(n)=3 h_{I Q 3}(t) \otimes a_{3}(n)+3 h_{I Q 3, i m}(t) \otimes a_{2}^{*}(n)
\end{gathered}
$$

Now the individual signal components can be easily obtained by some elementary manipulations, what need to do is estimating the responses and reconstructing a cancellation signal, then subtracting it from the SI in digital domain at last.

\section{Joint digital SI cancellation}

As discussed in section 2, the residual SI in the digital domain considering both IQ imbalance and PA nonlinear distortion has been analyzed. Different from [9], the equation about SI is expressed as the sum of six distrinct parts rather than four components. Next, the corresponding unknown coefficients will be estimated and then the final SI cancellation will be carried out. In Fig. 3, the structure of digital SI cancellation is depicted.
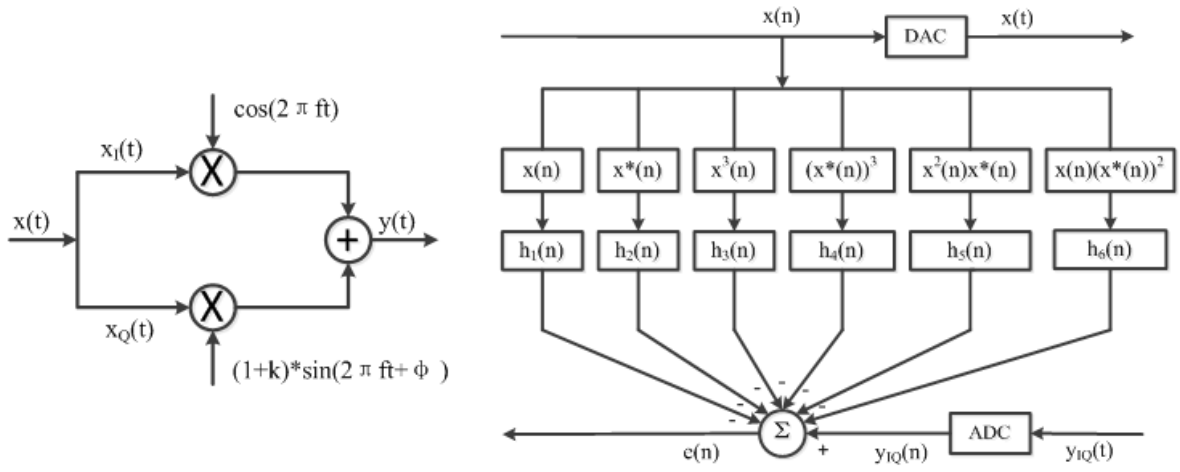

Fig. 2. The structure of quadrature modulator with IQ imbalance.

Fig. 3. A schematic diagram of the proposed joint digital SI cancellation.

From (16), $x(n)$ is the original signal in the baseband, $x^{*}(n)$ denotes the complex conjugate, $x^{3}(n)$ represents the third order non-linearity, and the others are the cross combination items of IQ imbalance and PA nonlinear distortion. Since the transmitted signal is known, the above six items are also available. It's assumed that the number of signal samples is $N$, then (16) can be rewritten with vector-matrix notation 


$$
\begin{aligned}
\boldsymbol{y}_{I Q} & =\boldsymbol{X} \boldsymbol{h}_{1}+\boldsymbol{X}^{*} \boldsymbol{h}_{2}+\boldsymbol{X}^{3} \boldsymbol{h}_{3}+\left(\boldsymbol{X}^{*}\right)^{3} \boldsymbol{h}_{4}+\left(\boldsymbol{X}^{2} \boldsymbol{X}^{*}\right) \boldsymbol{h}_{5}+\left(\boldsymbol{X}\left(\boldsymbol{X}^{*}\right)^{2}\right) \boldsymbol{h}_{6}+W \\
& =\left[\boldsymbol{X} \boldsymbol{X}^{*} \boldsymbol{X}^{3}\left(\boldsymbol{X}^{*}\right)^{3}\left(\boldsymbol{X}^{2} \boldsymbol{X}^{*}\right)\left(\boldsymbol{X}\left(\boldsymbol{X}^{*}\right)^{2}\right)\right]\left[\boldsymbol{h}_{1} \boldsymbol{h}_{2} \mathrm{~L} \boldsymbol{h}_{6}\right]^{T}+W \\
& =\boldsymbol{X}_{\text {aug }} \boldsymbol{h}_{\text {aug }}+W
\end{aligned}
$$

where $\boldsymbol{y}_{I Q}=\left[\begin{array}{llll}y_{I Q}(1) & y_{I Q}(2) & \cdots & y_{I Q}(N)\end{array}\right]^{T},[\cdot]^{T}$ indicates the transpose of vector and $W$ is the corresponding vector form of $w(n)$.

Here, the values of signal at further/before times than $n$ are used to estimate the value of signal at time $n$ [7]. For example, $\boldsymbol{X}$ is the Toeplitz matrix of $x(n)$

$$
\boldsymbol{X}=\left[\begin{array}{ccccc}
x(k) & \cdots & x(1) & \cdots & x(-k+1) \\
x(k+1) & \cdots & x(2) & \cdots & x(-k+2) \\
\vdots & \ddots & \vdots & \ddots & \vdots \\
x(N+k-1) & \cdots & x(N) & \cdots & x(-k+N)
\end{array}\right]
$$

where $k$ is the numbers of pre- and post-cursor filter taps. Similarly, the other signal matrix in (21) can also be written in the form of Toeplitz matrix and will not be enumerated.

The goal is to find the optimal estimation of the vector $\boldsymbol{h}_{\text {aug }}$, it can be calculated following the LS criterion

$$
\hat{\boldsymbol{h}}_{\text {aug }}=\left(\boldsymbol{X}_{\text {aug }}^{H} \boldsymbol{X}_{\text {aug }}\right)^{-1} \boldsymbol{X}_{\text {aug }} \boldsymbol{y}_{I Q}
$$

where $[\cdot]^{H}$ indicates the conjugate transpose of matrix. From the augmented channel estimation, the individual channel estimations can be directly obtained as follows

$$
\begin{gathered}
\hat{\boldsymbol{h}}_{1}=\left[\begin{array}{llll}
\hat{h}_{\text {aug }}(0) & \hat{h}_{\text {aug }}(1) & \cdots & \hat{h}_{\text {aug }}(2 k-1)
\end{array}\right]^{T} \\
\hat{\boldsymbol{h}}_{2}=\left[\begin{array}{lllll}
\hat{h}_{\text {aug }}(2 k) & \hat{h}_{\text {aug }}(2 k+1) & \cdots & \hat{h}_{\text {aug }}(4 k-1)
\end{array}\right]^{T}
\end{gathered}
$$

and

$$
\hat{\boldsymbol{h}}_{6}=\left[\begin{array}{lllll}
\hat{h}_{\text {aug }}(10 k) & \hat{h}_{\text {aug }}(10 k+1) & \cdots & \hat{h}_{\text {aug }}(12 k-1)
\end{array}\right]^{T}
$$

The equations of $\hat{\boldsymbol{h}}_{3} \sim \hat{\boldsymbol{h}}_{5}$ are omitted here.

As mentioned above, the cancellation signal $g_{D}(n)$ in Fig. 1 can be successfully constructed with good estimations of the responses of individual signal components. Thereby, we can eliminate it from the SI after RF cancellation to the level of noise floor as close as possible and render the residual SI negligible.

\section{Simulation results and analysis}

In this section, the validity of the above analysis results is verified with full waveform simulations of a complete full-duplex transceiver. A similar transceiver model, as shown in Fig. 1, will be implemented in MATLAB platform, and the IQ imbalance as well as the PA non-linearity will be both taken into consideration. 
Firstly, the whole simulation is implemented in a dual-antenna case at WiFi $2.4 \mathrm{GHz}$ with an average transmit power of $20 \mathrm{dBm}$ and a bandwidth of $10 \mathrm{MHz}$. The signal of interest and the noise floor is set to $-85 \mathrm{dBm}$ and $-100 \mathrm{dBm}$ separately. Besides, PA and quadrature modulator in transceiver chains are installed with typical parameters in practical systems. The adverse benefit of IQ imbalance is modeled by introducing some offsets both in amplitude $(k)$ and phase $(\phi)$ to I and Q branches, and the IRR is set to $30 \mathrm{~dB}$. Moreover, the channel is first estimated through the transmitted training samples, and then together with the signal samples, is subtracted from the SI. The performance of the simulation is analyzed mainly based on the metric: the cancellation of residual SI in digital domain.

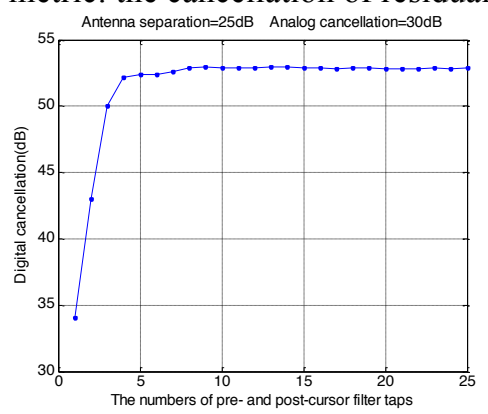

Fig. 4. The amount of digital cancellation obtained using the proposed canceller with different numbers of pre- and post-cursor filter taps

In the first simulation scenario, we investigate the relationship between the amount of digital cancellation and the value of $k$ by changing the number of pre- and post-cursor filter taps. Fig. 4 shows that the digital cancellation is improved with the increase of $k$ to some degree, but when $k$ is higher than 10 , there are no significant differences in the obtained digital cancellation. It suggests that the nearby signal having great correlation with the estimated signal, which then affects the accuracy of channel estimation, and the signals much further have little impact on it. Thus we should choose an appropriate number of filter taps for digital SI cancellation. In order to achieve the best performance, we fix the value of $k$ to 10 in the following simulations.

Next, four different cancellation schemes are compared considering both IQ imbalance and PA nonlinear distortion: classical linear canceller; linear canceller with image components, the channel response function can be estimated as $\hat{\boldsymbol{h}}_{\text {augl }}=\left(\boldsymbol{X}_{\text {augl }}^{H} \boldsymbol{X}_{\text {augl } 1}\right)^{-1} \boldsymbol{X}_{\text {augl } l}^{H} \boldsymbol{y}_{I Q}$, and $\hat{\boldsymbol{h}}_{3} \sim \hat{\boldsymbol{h}}_{6}=\mathbf{0}$, where $\boldsymbol{X}_{\text {augl }}=\left[\begin{array}{ll}\boldsymbol{X} & \boldsymbol{X}^{*}\end{array}\right]$ and $\hat{\boldsymbol{h}}_{\text {augl }}=\left[\begin{array}{ll}\hat{\boldsymbol{h}}_{1} & \hat{\boldsymbol{h}}_{2}\end{array}\right]^{T}$; nonlinear canceller with third order non-linearity, the channel can be estimated as $\hat{\boldsymbol{h}}_{\text {aug } 2}=\left(\boldsymbol{X}_{\text {aug } 2}^{H} \boldsymbol{X}_{\text {aug } 2}\right)^{-1} \boldsymbol{X}_{\text {aug } 2}^{H} \boldsymbol{y}_{I Q}, \quad$ and $\hat{\boldsymbol{h}}_{2}, \hat{\boldsymbol{h}}_{4} \sim \hat{\boldsymbol{h}}_{6}=\mathbf{0}$, where $\boldsymbol{X}_{\text {aug } 2}=\left[\begin{array}{ll}\boldsymbol{X} & \boldsymbol{X}^{3}\end{array}\right]$ and $\hat{\boldsymbol{h}}_{\text {aug } 2}=\left[\begin{array}{ll}\hat{\boldsymbol{h}}_{1} & \hat{\boldsymbol{h}}_{3}\end{array}\right]^{T}$; the proposed canceller. The measured power spectral densities (PSD) are shown in Fig. 5 and Fig. 6 respectively. 


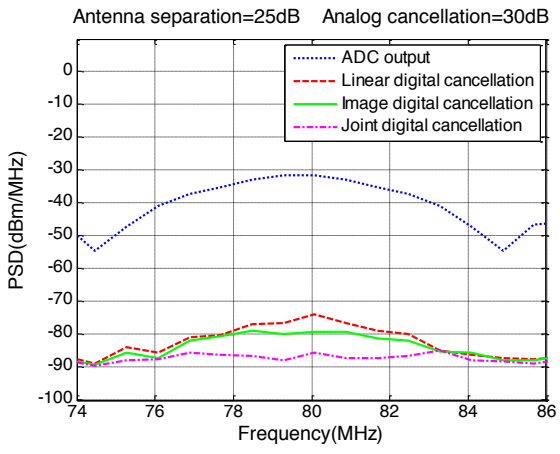

Fig. 5. The achieved power spectral densities with three different cancellers respectively, compared with ADC output.

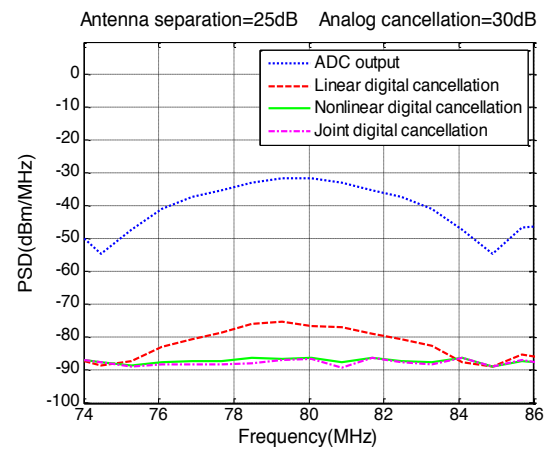

Fig. 6. The achieved power spectral densities with three different cancellers respectively, compared with ADC output.

The analysis results show that: the performance of the proposed canceller is superior to the other three cancellers. The linear digital canceller can attenuate the SI signal only by $45 \mathrm{~dB}$ after RF cancellation, while the linear canceller with image components and the nonlinear canceller with third order non-linearity achieve $47 \mathrm{~dB}$ and $52 \mathrm{~dB}$ at the frequencies from $75 \mathrm{MHz}$ to $85 \mathrm{MHz}$ respectively. Intuitively, the proposed canceller obtained the best performance up to $53 \mathrm{~dB}$. To summarize, the simulation results prove the validity of the proposed model foregoing. Meanwhile, it illustrates clearly that only eliminating the linear components is not enough, the effects of RF impairments must be taken into consideration and attenuated in the digital domain. Although the joint digital cancellation scheme increases the complexity of the model and computation to some extent, it does improve the interference cancellation ratio in the digital domain. Combined with antenna separation and analog cancellation, the overall amount of cancellation can be the same level as $108 \mathrm{~dB}$.

In Fig. 7, the whole amounts of SI attenuation of above four schemes under different signal-to-noise ratios (SNR) are presented. The result of (a) shows that when SNR is increased, the PA non-linearity becomes the dominant noise component. The difference between (a) and (b) is that $I R R=30 \mathrm{~dB}$ in (a) and $I R R=25 \mathrm{~dB}$ in (b). It can be observed that the distances between the upper two lines and the lower two lines are both little bigger than these in (a) as SNR is increased. That is to say, with the increasing of the IQ imbalance, the advantage of the joint digital cancellation is more prominent.

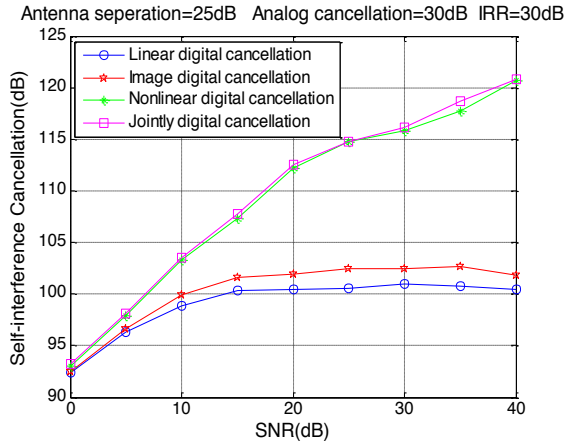

(a)

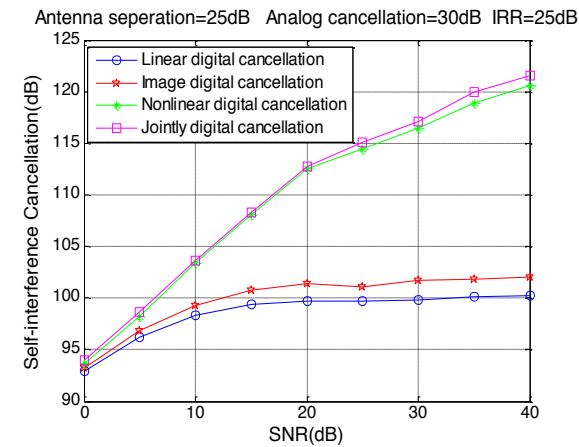

(b)

Fig. 7. The amount of SI cancellation as a function of SNR under the circumstance of $25 \mathrm{~dB}$ antenna suppression and $30 \mathrm{~dB}$ analog cancellation, with (a) $I R R=25 \mathrm{~dB}$ and (b) $I R R=30 \mathrm{~dB}$. 


\section{Conclusion}

In this paper, a joint digital SI cancellation scheme for full-duplex radios is proposed, and the adverse benefits of IQ imbalance and PA non-linearity are analyzed in detail. The proposed canceller increases the amount of cancellable SI signal power up to $108 \mathrm{~dB}$ by taking all the distortions caused by IQ imbalance and PA non-linearity into account rather than partially, which means that it's able to suppress the SI to the next significant noise component. Accordingly, we will consider the LNA non-linearity together in the cancellation model to make the residual SI signal equal or even lower than the noise floor in the future.

\section{References}

1. D.W. Bliss, P.A. Parker, A.R. Margetts, IEEE/SP 2 , 478(2007).

2. M. Duarte, A. Sabharwal, IEEE. ASILOMAR 3, 1558 (2010).

3. JI. Choi, M. Jain, K. Srinivasan, P. Levis, S. Katti, ACM MOBICOM 49, 1 (2010).

4. A. Sabharwal, P. Schniter, D. Guo, D.W. Bliss, S. Rangarajan, R. Wichman, IEEE. Journ. Sel. Areas Commun. 32, 1637 (2014).

5. M. Duarte, C. Dick, A. Sabharwal, IEEE. Trans. Wirel. Commun. 11, 4296 (2012).

6. Y. Sun, H. Zuo, L. Song, Journ. Electr. Sci. Tech. 45, 873 (2016).

7. D. Bharadia, E. Mcmilin, S. Katti, ACM SIGCOMM. 43, 375 (2013).

8. T. Chen, S. Liu, IEEE. GLOBECOM 1 (2015).

9. D. Korpi, L. Anttila, et al, IEEE. Journ. Sel. Areas Commun. 32, 1674 (2014).

10. L. Samara, M. Mokhtar, O. Ozdemir, et al, IEEE. Commun. Lett. 21, 314 (2017).

11. M. Sakai, H. Lin, K. Yamashita, Phys. Commun. 18, 2 (2016).

12. A. Masmoudi, T. Le-Ngoc, IEEE. Trans. Vehi. Tech. 65, 5122 (2017).

13. E. Ahmed, A.M. Eltawil, A. Sabharwal, IEEE. Asilom. Confer. Sig. Syst. Comput. 118, 1199 (2013).

14. L. Anttila, D. Korpi, V. Syrjala, M. Valkama, IEEE. Asilom. Confer. Sig. Syst. Comput. 118, 1193 (2013).

15. L. Anttila, M. Valkama, M. Renfors, IEEE. Trans. on Circ. \& Syst. II Expr. Bri. 55, 359 (2008).

16. S. Hu, M. Li, Acad. Semin. Commun. Depart. Chin. Univ. (2009).

17. J. Lu, Modern Radar 21,100 (1999). 